\title{
Recherche universitaire et priorités nationales : l'effet du financement public sur la recherche en énergie solaire au Canada
}

\author{
ROBERT DALPÉ* et YVES GINGRAS†
}

\section{RÉSUMÉ}

Nous étudions l'impact des deux principales sources de financement de la recherche universitaire dans le domaine de l'énergie solaire (le Programme des actions thématiques en énergie du CRSNG et les programmes du ministère de l'Énergie, des Mines et des Ressources), afin de déterminer si elles ont orienté la recherche en fonction des priorités gouvernementales. La méthodologie consiste à établir une relation entre les intrants (le financement) et les extrants (les publications scientifiques). La relation la plus forte unit les chercheurs ayant publié dans les revues spécialisées en énergie solaire et ceux subventionnés par le CRSNG. Au début de la période étudiée, à la fois le niveau des publications et celui des subventions ont augmenté considérablement. On remarque l'émergence d'un noyau de chercheurs en énergie solaire, qui a eu la capacité d'obtenir du financement de plusieurs sources.

\footnotetext{
ABSTRACT

The role of the two main sources of university research financing in the area of solar energy (the Strategic Grants of the NSERC and the solar energy Programs of Energy, Mines and Ressources) is examined in order to assess whether they oriented research in the direction of government programs. The applied methodology aims at establishing a relationship between inputs (financing) and outputs (scientific publications). The strongest ties seem to bind researchers

*Département de science politique, Université de Montréal, et Centre de recherche en développement industriel et technologique (CREDIT), UQAM

$\nmid$ Département d'histoire et Centre de recherche en développement industriel et technologique (CREDIT), UQAM

Nous remercions Frances Anderson, Élaine Gauthier, Luc Bélanger et Jean Guay pour leur contribution à la réalisation de cette recherche, qui a bénéficée d'un financement du Fonds Cafir de l'Université de Montréal et d'Énergie, Mines et Ressources (Robert Dalpé), de même que du CRSH et du PAFACC de l'UQAM (Yves Gingras). Une version préliminaire de ce texte a été présentée au congrès de l'Association canadienne de science politique, Québec, juin 1989.
} 
having published in journals specialized in solar energy with those financed by NSERC. During the first years of the period under study, the number of publications and subsidies have both considerably increased. A scientific community on solar energy emerges and acquires the capacity to tap several different sources of financing.

Le gouvernement canadien finance la recherche universitaire en sciences naturelles principalement par le biais des programmes du Conseil de recherche en sciences naturelles et en génie (CRSNG). L'allocation des subventions se fait par un processus d'évaluation par les pairs. Ce contrôle collégial de la qualité des projets fait en sorte que la communauté des chercheurs fixe elle-même ses priorités et détermine ainsi, sans contraintes externes, l'allocation des fonds mis à sa disposition par les pouvoirs publics. Au cours des dix dernières années, ce modèle de la « république de la science» a fait l'objet de nombreuses critiques. Plusieurs études considèrent en effet que le processus d'évaluation par les pairs ne parvient pas à bien estimer la pertinence sociale des recherches et est peu sensible à l'émergence de problèmes nécessitant une intervention rapide pour répondre à de nouvelles prioritês socio-économiques (Roy, 1984 ; Irvine, Martin et Oldham, 1983). Pour contourner cette autonomie, jugée trop grande, de la communauté scientifique, les gouvernements ont été amenés à mettre sur pied de nouveaux programmes conçus spécifiquement pour orienter les activités de recherche des universitaires en fonction de leurs objectifs nationaux (Chapman et Farina, 1981 et 1983 ; Ford, Green, Steward et Ahmad, 1986). Pour être efficaces, ces nouveaux programmes devaient évaluer les demandes de façon à ce que les sujets de recherche retenus ne soient plus fixés en fonction des objectifs propres aux communautés scientifiques, mais plutôt en fonction de priorités gouvernementales prédéfinies.

L'énergie étant devenue, au milieu des années 70 , une des priorités du gouvernement canadien, les chercheurs universitaires ont eu accès à deux nouvelles sources de fonds. La première, le Programme des Actions thématiques du CRSNG, créé en 1977, visait la recherche dite «stratégique » pour développer une base de connaissances susceptible de servir à la solution de problèmes pratiques actuels ou prévisibles (selon la définition de Ford et al, 1986). La deuxième comprend toute une série de programmes gérés par la Division de l'énergie du Conseil national de recherches et par la Direction des économies d'énergie et des énergies renouvelables au ministère de l'Énergie, des Mines et des Ressources (EMR). Soumis à la politique d'impartition du gouvernement fédéral, ces programmes octroyaient, sous des formes variées, des contrats, à l'industrie surtout, mais également à des chercheurs universitaires.

Dans le cadre de cet article, nous étudierons le rôle de ces deux nouvelles sources de financement de la recherche universitaire sur le développement des activités dans le domaine de l'énergie solaire. Notre objectif est de voir dans quelle mesure ces deux programmes orientent la recherche universitaire en fonction des 
priorités gouvernementales. Plus spécifiquement, nous analyserons comment les scientifiques et les ingénieurs ont réagi face à ces programmes pour en connaître l'impact sur la dynamique de la communauté scientifique canadienne. Pour ce faire, nous analyserons les caractéristiques des clientèles des deux programmes et la production scientifique dans le domaine de l'énergie solaire. Après avoir présenté la méthodologie de la recherche, nous rappellerons brièvement les caractéristiques des sources de financement, pour analyser ensuite leurs clientèles et la production scientifique des chercheurs.

Chapman et Farina (1983) ont étudié l'impact, durant leurs trois premières années d'existence, des programmes thématiques du CRSNG sur l'attribution des fonds aux chercheurs canadiens. Leur principale conclusion était que ce nouveau programme avait eu peu d'effet : les chercheurs financés étaient ceux qui étaient auparavant soutenus par les programmes généraux du CRSNG. Leur analyse des contrats de recherche octroyés par d'autres ministères a aussi montré que la clientèle de ces programmes était différente de celle des programmes thématiques du CRSNG. Notre étude porte sur les dix années d'existence du programme et permet ainsi de mieux mesurer son effet à long terme. De plus, nous analysons également les subventions octroyées par EMR au cours de la même période. Enfin, notre approche diffère de celle de ces auteurs en ce qu'elle mesure l'impact de ces programmes sur la production de connaissances alors que Chapman et Farina s'intéressaient surtout aux relations entre les chercheurs subventionnés par le CRSNG et ceux qui obtenaient des contrats des ministères.

\section{MÉTHODOLOGIE}

La création de ces deux sources de financement de la recherche universitaire visait essentiellement deux objectifs: orienter le travail d'un certain nombre de scientifiques vers les recherches en énergie solaire et favoriser des recherches plus appliquées pouvant contribuer directement à l'amélioration des technologies solaires commercialisées par l'industrie. Pour atteindre ces objectifs, le gouvernement offrait un financement spécial. Cette offre devait entraîner un accroissement de la recherche dans le domaine de l'énergie solaire par le biais d'un encouragement accru aux chercheurs œuvrant déjà dans le secteur et en incitant des chercheurs à réorienter leurs activités sur des problèmes reliés à l'énergie solaire.

Pour évaluer l'effet de ces programmes sur les activités de recherche dans le domaine de l'énergie solaire, nous allons tenter de répondre à la question suivante : dans quelle mesure les chercheurs subventionnés ont-ils effectivement contribué au développement des connaissances sur l'énergie solaire? En prenant pour indicateur l'évolution de la production scientifique canadienne dans ce domaine au cours de la période 1974-1987, et en identifiant les caractéristiques des chercheurs, on pourra identifier la clientèle qui a eu recours à ces programmes et relier la croissance des activités de recherche à celle des fonds offerts par les différents organismes gouvernementaux. 
La production canadienne en énergie solaire sera ici mesurée à partir de l'inventaire des plus importantes revues internationales en énergie solaire. Nous avons retenu huit revues d'après la liste constituée par Leydesdorff et van der Schaar (1987), à partir d'entrevues auprès des chercheurs hollandais en énergie solaire. Cette liste comprend d'abord six revues spécialisées (Solar Cells, Solar Energy, Solar Energy Materials, Applied Energy, Energy Conversion, et International Journal of Energy Research). Plusieurs autres revues (en particulier les revues de physique appliquée, physique de l'état solide, électrochimie et de transferts de chaleur) publient également des travaux en énergie solaire. À la suite de Leydesdorff et van der Schaar, nous avons retenu les deux qui publiaient les articles le plus directement axés sur le solaire, soit Journal of Applied Physics et Journal of the Electrochemical Society. Ces huit revues constituent les principaux lieux de publication et couvrent la plus grande partie de la production en énergie solaire. Bien que les chercheurs canadiens puissent publier des articles qui nous échappent, il est probable que la plupart de ces articles ne porteront qu'indirectement sur l'énergie solaire. L'absence de chercheurs de notre liste ne signifie donc pas qu'ils ne publient pas, mais que leur production concerne plutôt des domaines connexes au solaire ou encore relèvent de la recherche fondamentale.

Le dépouillement des huit revues nous permet d'établir la liste des chercheurs canadiens publiant en énergie solaire et de tracer un profil de la communauté canadienne. Une première méthode permettant de relier cette production aux organismes subventionnaires consiste à examiner les remerciements où sont souvent mentionnées les subventions obtenues pour réaliser les recherches rapportées dans les articles. Dans leur étude du financement de la recherche en biotechnologie, Rip et Hennekan (1985) ont déjà montré qu'il s'agit là d'une source intéressante pour retracer les organismes subventionnaires. Cet instrument nous permettra d'établir un profil des organismes subventionnaires en énergie solaire et de situer la place des programmes dont nous cherchons à évaluer l'impact. En croisant la liste des chercheurs subventionnés avec celle des chercheurs qui publient, on pourra déterminer si ceux qui publient sont aussi ceux qui sont financés par nos deux organismes.

\section{LES PROGRAMMES DE FINANCEMENT}

\section{L'importance de l'énergie solaire dans le programme des subventions thématiques du CRSNG}

Le CRSNG subventionne les chercheurs universitaires via ses programmes réguliers. En 1977, des programmes thématiques ont été ajoutés en vue d'orienter le travail des chercheurs dans des secteurs jugés d'importance nationale, dont celui de l'énergie. Alors que les programmes réguliers visent des individus, les programmes thématiques visent des projets. Ainsi, la priorité est accordée aux universitaires dont les recherches portent sur des problèmes pertinents, qui correspondent aux besoins de la situation énergétique canadienne, dans le but d'améliorer l'expertise dans le domaine, de contribuer à la formation d'une 
Recherche universitaire et priorités nationales: l'effet du

Tableau 1

Subventions du programme thématique du CRSNG dans le domaine de l'énergie (Milliers de \$)

\begin{tabular}{|c|c|c|c|c|c|c|c|c|c|}
\hline année & $\begin{array}{l}\text { Budget } \\
\text { total (A) }\end{array}$ & $\begin{array}{l}\text { Subv. thema- } \\
\text { tiques (B) }\end{array}$ & $\begin{array}{l}\mathrm{B} / \mathrm{A} \\
(\%)\end{array}$ & $\begin{array}{r}\text { Éncrgic } \\
\text { (C) }\end{array}$ & $\begin{array}{l}\mathrm{C} / \mathrm{B} \\
(\%)\end{array}$ & Nombre & $\begin{array}{c}\text { Solaire } \\
\text { (D) }\end{array}$ & $\begin{array}{l}\mathrm{D} / \mathrm{C} \\
(\%)\end{array}$ & Nombre \\
\hline $1977-78$ & 97,690 & 2,346 & $(2.4)$ & 1,072 & $(45,7)$ & 35 & 190 & $(17.7)$ & 7 \\
\hline $1978-79$ & 109,706 & 7,356 & $(6.7)$ & 3,884 & (52.8) & 96 & 563 & (14.5) & 17 \\
\hline $1979-80$ & 118,400 & 10,707 & $(9.0)$ & 3,726 & (34.8) & 99 & 606 & $(15.1)$ & 20 \\
\hline $1980-81$ & 158,950 & 17,780 & (11.2) & 6,066 & (34.1) & 145 & 954 & $(15.6)$ & 26 \\
\hline $1981-82$ & 196.944 & 21.565 & (10.9) & 6.539 & (30.3) & 127 & 1,074 & (16.4) & 20 \\
\hline $1982-83$ & 238,702 & 26,305 & (11.0) & 7,440 & (28.3) & 120 & 1,551 & $(20.8)$ & 16 \\
\hline $1983-84$ & 274,621 & 28,256 & $(10.3)$ & 7,824 & $(27.7)$ & 122 & 1,388 & $(17.7)$ & 14 \\
\hline $1984-85$ & 300,528 & 32,254 & $(10.7)$ & 4,854 & $(15.0)$ & 98 & 1,225 & $(25.2)$ & 11 \\
\hline $1985-86$ & 298,976 & 32.481 & $(10.9)$ & 3,751 & (11.5) & 70 & 345 & (9.1) & 6 \\
\hline $1986-87$ & 308,065 & 31.964 & $(10.4)$ & 3,589 & $(11.2)$ & 64 & 166 & $(4.6)$ & 5 \\
\hline
\end{tabular}

Source: donnćes compilées à partir des rapports annuels.

main-d'œuvre spécialisée et d'accélérer le développement des technologies. Le programme subventionne la recherche appliquée, mais aussi la recherche fondamentale dans la mesure où il s'agit de travaux pertinents par rapport au domaine de l'énergie.

Le programme des subventions thématiques constitue « le plus grand effort du Conseil dans les domaines scientifiques choisis par ce dernier plutôt que par les chercheurs ". Alors que les programmes ordinaires ne sont évalués que par les pairs, les programmes thématiques ont un caractère plus appliqué et les comités de sélection comprennent des membres provenant du milieu industriel et des organismes gouvernementaux. Le programme de l'énergie est d'ailleurs celui qui contient la plus grande proportion de membres provenant du milieu industriel (CRSNG, 1985, 62). Cependant, le Conseil admet que « les thèmes choisis, comme l'énergie, ne sont définis que dans les grandes lignes » (CRSNG, 1985, 60) ce qui laisse une bonne marge de liberté aux scientifiques.

Comme l'indique le tableau 1, la part du budget du CRSNG consacrée aux subventions thématiques oscille autour de $11 \%$. Au cours des années, le nombre de domaines jugés prioritaires est passé de 3 (1977) à 8 (1984), mais le domaine de l'énergie, qui voit sa part diminuée au fil des ans, demeure le plus important du programme.

Dans l'ensemble des filières énergétiques subventionnées par le CRSNG, l'énergie solaire occupe une place importante. S'accaparant 16,3\% du budget total pour la période 1977-1986, elle vient au premier rang de l'ensemble des filières énergétiques et passe au second rang pour le nombre total de projets subventionnés avec 13,9\% de l'ensemble (tableau 2). À l'exception de la fusion, de la conservation d'énergie et du stockage qui reçoivent respectivement $15,3 \%, 12,6 \%$ et $11,8 \%$ du montant global pour la même période, les autres filières reçoivent moins de $7 \%$ des sommes dépensées par le Conseil.

De 1977-1978 à 1986-1987, 37 chercheurs principaux, associés à 46 co-chercheurs (dont 2 sont aussi chercheurs principaux dans d'autres projets), ont obtenu des subventions pour faire de la recherche en énergie solaire. Les sommes 
Tableau 2

Filières éncrgétiques subventionnćes par le CRSNG $1977-78$ a $1986-87$

\begin{tabular}{lrrr}
\hline Filières & Nombre de projets & $\begin{array}{r}\text { Subventions } \\
\text { (Milliers } \$ \text { ) }\end{array}$ & $\%$ \\
\hline Énergie solaire & 57 & 8,366 & 16.3 \\
Fusion & 40 & 7,863 & 15.3 \\
Conservation & 56 & 6,481 & 12.6 \\
Stockage & 60 & 6,032 & 11.8 \\
Charbon & 32 & 3,482 & 6.8 \\
Biomasse & 29 & 3,461 & 6.7 \\
Nouveaux carburants & 25 & 3,168 & 6.2 \\
Sables pétroliferes et lourds & 19 & 2,423 & 4.7 \\
Exploration & 14 & 1,987 & 3.9 \\
Fission & 17 & 1,737 & 3.4 \\
Autres & 62 & 6,330 & 12.3 \\
Total & 411 & 51,330 & 100.0
\end{tabular}

Source: données compilées à partir des rapports annuels.

octroyées annuellement ont augmenté jusqu'en 1982-1983, pour connaître une forte diminution en 1984-1985, année où le gouvernement fédéral, aux prises avec un important déficit budgétaire, a coupé de moitié les fonds consacrés à la recherche sur les énergies renouvelables (Gingras et Rivard, 1988) (Tableau 1).

Le Tableau 3 indique l'affiliation départementale des chercheurs, ce qui nous permet de voir que les travaux en énergie solaire subventionnés par le CRSNG sont surtout le fait de chimistes et de physiciens. La position secondaire des ingénieurs suggère que les travaux entrepris sont davantage à l'étape de la recherche fondamentale ou appliquée que du développement.

La plus importante source de fonds pour les chercheurs universitaires demeure les subventions ordinaires du CRSNG. Tous les chercheurs qui ont bénéficié des subventions thématiques en énergie avaient auparavant obtenu des subventions ordinaires. Le montant des subventions thématiques est toutefois plus élevé : le montant de la subvention thématique obtenue la première année est en moyenne le double des fonds provenant de leur comité disciplinaire. Sur 37 chercheurs, 32 ont eu davantage de fonds du programme thématique que du programme ordinaire. Pendant la durée de la subvention thématique (normalement trois ans), les chercheurs bénéficient donc de montants beaucoup plus importants.

\section{Les contributions d'EMR à la recherche en énergie solaire}

Les principaux programmes en énergie solaire ont été gérés par le CNR jusqu'en 1985, date à laquelle la Division de l'énergie a été fermée et les programmes transférés à la Direction des économies d'énergie et des énergies renouvelables d'EMR (CNR, 1986). Les grands objectifs de ces programmes sont, première- 
Tableau 3

Appartenance disciplinaire des chercheurs universitaires en énergic solaire

\begin{tabular}{|c|c|c|c|c|c|c|c|c|}
\hline \multirow[t]{2}{*}{ Disciplin } & \multicolumn{3}{|c|}{$\begin{array}{l}\text { Subventionnés par } \\
\text { le CRSNG }\end{array}$} & \multicolumn{3}{|c|}{ Financés par EMR } & \multicolumn{2}{|c|}{ Publications } \\
\hline & $\begin{array}{l}\text { Obtenant } \\
\text { de } 20000\end{array}$ & $10+$ & $\begin{array}{r}\text { Tous lcs } \\
\text { chercheurs }\end{array}$ & $\begin{array}{l}\text { Obtenant } \\
\text { de } 200.000\end{array}$ & + & $\begin{array}{l}\text { Tous les } \\
\text { ercheurs }\end{array}$ & $\begin{array}{l}\text { Au moins } \\
3 \text { articles }\end{array}$ & $\begin{array}{l}\text { Tous les } \\
\text { chercheur }\end{array}$ \\
\hline \multicolumn{2}{|c|}{ Chimie } & 4 & 12 & & 0 & 0 & 3 & 29 \\
\hline \multicolumn{2}{|c|}{ Physique } & 4 & 8 & & 0 & 4 & 4 & 19 \\
\hline \multicolumn{2}{|c|}{ Génie électrique } & 0 & 7 & & 0 & 0 & 5 & 25 \\
\hline \multicolumn{2}{|c|}{ Génie mécanique } & 0 & 5 & & 3 & 7 & 5 & 30 \\
\hline \multicolumn{2}{|c|}{ Autre* ou inconnu } & 2 & 5 & & 3 & 6 & 5 & 51 \\
\hline \multicolumn{2}{|c|}{ Total } & 10 & 37 & & 6 & 17 & 22 & 154 \\
\hline
\end{tabular}

* autre discipline ou membres de centres de recherche n'ayant pas d'affiliation disciplinaire.

Sources: CRSNG: données compilées à partir des rapports annucls; EMR: données compilées à partir du Bulletin Recherche el développement: publications: à partir des huit revues en solaire.

ment, d'établir les bases techniques nécessaires à la mise au point d'une technologie fiable et rentable; deuxièmement, de contribuer à la création de produits et de procédés qui présentent des possibilités commerciales à court terme et, troisièmement, de contribuer à la mise au point d'une nouvelle technologie d'approvisionnement énergétique à moyen et long termes.

Les premiers programmes ont été adoptés en 1978, pour une période de cinq ans, et $\$ 125$ millions étaient disponibles bien que seulement un cinquième de cette somme ait été dépensé. PASEM (Program for Assistance to Solar Energy Manufacturers), visait l'amélioration des produits et le développement des capacités de production, alors que le second, PUSH (Purchase and Use of Solar Heating), concernait l'achat de systèmes solaires par le gouvernement fédéral (Berkowitz, 1978; CNR, 1986). Ces deux programmes s'adressaient à une clientèle industrielle. En 1983, un programme de recherche-développement et démonstration en énergie solaire d'environ $\$ 10$ millions par année a été créé. En 1984, lors de son transfert à EMR, le programme est devenu le Programme de développement de l'énergie solaire. Son objectif était de permettre le développement de technologies et d'entreprises pour qu'à la fin du programme, en 1988, l'industrie soit en mesure de commercialiser ses produits sans le soutien du gouvernement. Parallèlement à ces programmes visant surtout le développement de produits directement utilisables, quelques programmes plus généraux de recherche et de développement s'adressaient à l'ensemble de la communauté des chercheurs. Par ailleurs, dans le cadre d'autres programmes du subvention, le CNR et EMR ont aussi octroyé quelques contrats de recherche dans le domaine de l'énergie solaire.

Une autre intervention importante a été le financement de cinq «centres d'expertise », qui offrent des services à l'industrie solaire (Enermodal Engineering, 1987). Quatre de ces centres se trouvent dans les universités, le cinquième étant à l'Ontario Research Foundation. À l'Université de Waterloo, on retrouve le 
Tableau 4

Évolution du financement offert par EMR

\begin{tabular}{|c|c|c|c|c|c|c|}
\hline \multirow{3}{*}{$\begin{array}{l}\text { Annee } \\
\text { Financement }\end{array}$} & \multicolumn{4}{|c|}{ En solaire } & \multicolumn{2}{|c|}{ Tous les domaines } \\
\hline & \multicolumn{2}{|c|}{ Universitaire } & \multicolumn{2}{|c|}{ Total } & \multirow[b]{2}{*}{ Projets } & \multirow[b]{2}{*}{ (Milliers \$) } \\
\hline & Projets & $\begin{array}{l}\text { Financement } \\
\text { illiers\$) }\end{array}$ & Projets & $\begin{array}{l}\text { Financement } \\
\text { (Milliers \$) }\end{array}$ & & \\
\hline $1977-1978$ & 1 & 19 & 5 & 127 & 44 & 1138 \\
\hline $1978-1979$ & 1 & 6 & 5 & 300 & 39 & 1445 \\
\hline $1979-1980$ & 0 & 0 & 4 & 46 & 38 & 1506 \\
\hline 1980.1981 & 0 & 0 & 0 & 0 & 25 & 1080 \\
\hline $1981-1982$ & 0 & 0 & 0 & 0 & 37 & 3889 \\
\hline $1982-1983$ & 1 & 245 & 5 & 276 & 65 & 8026 \\
\hline $1983-1984$ & 0 & 0 & 3 & 80 & 83 & 9757 \\
\hline $1984-1985$ & 0 & 0 & 7 & 144 & 124 & 13817 \\
\hline $1985-1986$ & 2 & 584 & 44 & 2391 & 135 & 12706 \\
\hline $1986-1987$ & 12 & 1937 & 71 & 4803 & 194 & 32809 \\
\hline $1987 \cdot 1988$ & 3 & 555 & 49 & 2451 & 169 & 11543 \\
\hline Total: & 20 & 3346 & 193 & 10168 & 953 & 98316 \\
\hline
\end{tabular}

Source: donnces compilćes à partir du Bulletin Recherche el dévelopoement.

Watsun Simulation Laboratory, qui fournit un support aux utilisateurs et des programmes d'amélioration par la simulation par ordinateur des performances de divers capteurs, et le Solar Thermal Research Laboratory, qui effectue de la recherche appliquée sur les systèmes de collecteurs d'énergie. Le Solar Calorimetry Laboratory de l'Université Queen's développe des méthodes pour évaluer le rendement des différents types de collecteurs d'énergie solaire. Finalement, à l'Institut national de la recherche scientifique (INRS-énergie), les chercheurs, en collaboration avec les entreprises privées, mettent au point divers produits.

Nous retenons ici uniquement les contrats octroyés à des universitaires par la Direction des économies d'énergie et des énergies renouvelables d'EMR, ce qui comprend les recherches régulières sur la question depuis 1977, en plus des programmes gérés à partir de 1985.

De 1977-1978 à 1987-1988, la Direction des économies d'énergie et des énergies renouvelables d'EMR a attribué 953 contrats de recherche totalisant 98,3 M\$. De ce nombre, 193 portent sur l'énergie solaire, pour un montant de $10,2 \mathrm{M} \$$ (soit 10,8\% du budget) (tableau 4). Les trois autres secteurs ayant reçu le plus de subventions sont ceux de la conservation $(38,5 \%)$, de la biomasse $(22,4 \%)$ et du pétrole et du gaz $(11,4 \%)$ (tableau 5). Cette Direction subventionne assez peu les recherches en énergie solaire avant 1984, alors que les principaux programmes relèvent du CNR. Pendant les trois dernières années, le solaire va devenir une préoccupation importante puisque $33 \%$ des contrats et $17 \%$ du financement concernent ce domaine.

Les contrats octroyés par cette Direction d'EMR connaissent une augmentation jusqu'en 1986-1987. Contrairement au montant des subventions du CRSNG, qui chute après 1984, le programme d'EMR n'est pas touché par les réductions des budgets de recherche en énergie qui affectent durement les énergies renouvelables 
Tableau 5

Filières énergétiques financées par $E M R$

\begin{tabular}{lrrrrr}
\hline Filiezres & \multicolumn{3}{c}{ Universitaires } & \multicolumn{3}{c}{ Tous les projets } \\
& Milliers \$ & Nombre & Milliers \$ & Nombre & AVB (\%) \\
& $(\mathrm{A})$ & & $(\mathrm{B})$ & & \\
\hline Conservation & 1,496 & 18 & 37,811 & 186 & 4.0 \\
Solaire & 3,346 & 20 & 10,618 & 193 & 31.5 \\
Biomasse & 2,067 & 35 & 22,046 & 214 & 9.4 \\
Energic Eolienne & 0 & 0 & 1,809 & 26 & 0 \\
Autre energic renouvelable & 691 & 8 & 5.262 & 79 & 13.1 \\
Plusieurs energies renouv. & 103 & 5 & 1.774 & 41 & 5.8 \\
Pétrole et gaz & 705 & 9 & 11.251 & 108 & 6.3 \\
Autres ou non identifiable & 218 & 11 & 7.745 & 106 & 2.8 \\
Total & 8.627 & 106 & 98.316 & 953 & 8.8
\end{tabular}

Source: données compilées à partir du Bulletin Recherche et dévelopoemenl.

à la suite de l'élection du gouvernement conservateur en 1984 et de la réduction des prix du pétrole en 1985. Ce fait s'explique en partie par les engagements pris avant 1984 et par la fermeture de la Division des énergies au CNR qui entraîne le transfert de certains programmes vers EMR.

En solaire, 17 chercheurs universitaires, reçurent $3,3 \mathrm{M} \$$ pour réaliser 20 projets, ce qui représente $31,5 \%$ du financement consenti à l'énergie solaire. Un seul chercheur, qui a obtenu des contrats sur des aspects non techniques de l'énergie solaire, ne fait pas partie du secteur des sciences naturelles. Les chercheurs universitaires sont ici en concurrence avec l'industrie, qui avec les laboratoires gouvernementaux se partagent $68,5 \%$ des fonds alloués. Les chercheurs universitaires participent beaucoup moins aux autres domaines: ils obtiennent seulement $4,0 \%$ du financement en conservation et $9,4 \%$ en biomasse.

Comme l'indique le tableau 3, les subventions d'EMR vont surtout aux départements de génie, bien que la physique occupe encore une place de choix. On voit donc déjà une différence marquée entre les subventions du CRSNG et celles d'EMR : alors que les premières semblent subventionner davantage les travaux de recherche appliquée ou même fondamentale, les secondes privilégient des travaux plus près du stade du développement, comme l'indique l'importance de la présence de départements de génie mécanique, lesquels étaient à peu près absents du programme du CRSNG.

Il est intéressant de noter que les chercheurs qui ont obtenu le plus de financement d'EMR sont ceux qui font partie des centres d'expertise mentionnés plus haut. En effet, cinq des six chercheurs ayant obtenu plus de 200000 \$ en contrats de recherche appartiennent à un de ces centres, une partie de ces subventions servant d'ailleurs au fonctionnement des centres.

\section{Les clientèles}

Les programmes d'EMR et du CRSNG ont en partie les mêmes clientèles : 5 chercheurs ayant obtenu des contrats d'EMR sont aussi subventionnés par le 
Fig̣ure 1

Liens entre les subventions et les publications (nombre de chercheurs)

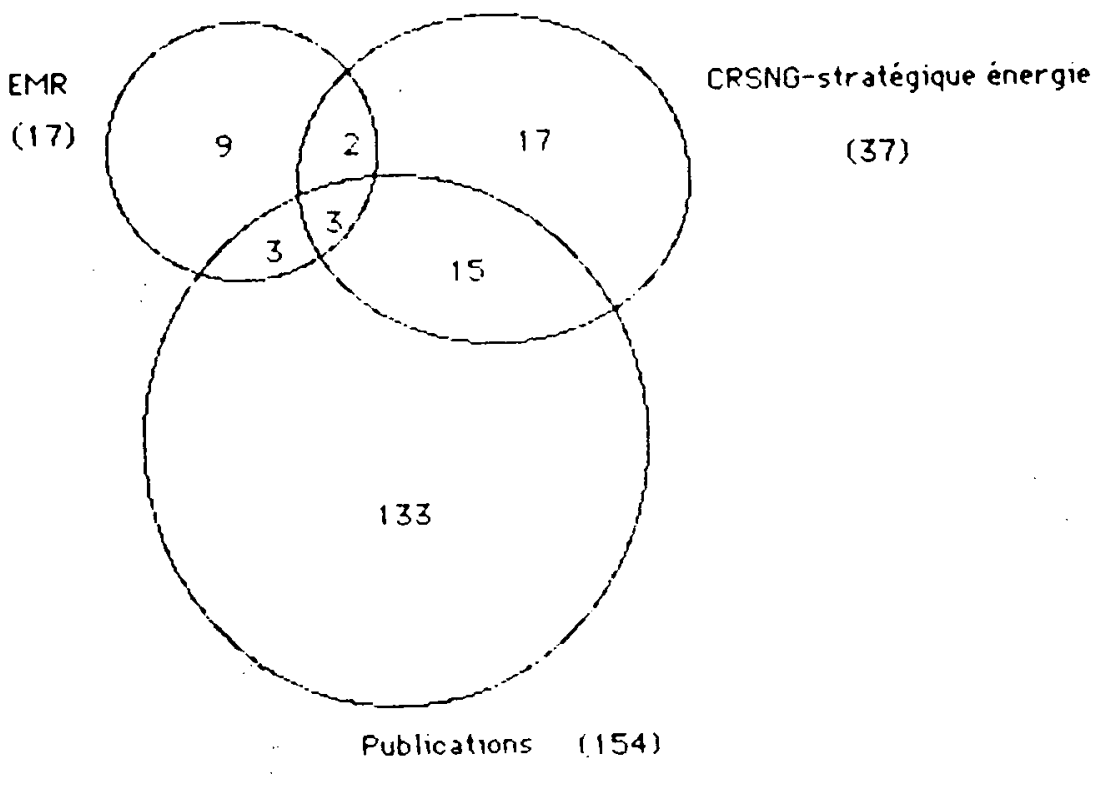

CRSNG (Figure 1). Dans le sous-groupe des chercheurs très subventionnés par les deux programmes, il existe cependant moins de liens: seulement 1 des 10 chercheurs très subventionnés (plus de 200000 \$) par le CRSNG a aussi obtenu des fonds d'EMR et seulement 2 des 6 équipes subventionnées par EMR ont obtenu des subventions du CRSNG (tableau 6). En ce qui concerne les 46 cochercheurs du CRSNG, 2 ont aussi obtenu des contrats d'EMR.

Environ le tiers des chercheurs subventionnés par EMR ont aussi obtenu des subventions thématiques du CRSNG. Ces données suggèrent qu'il existe des liens entre les deux programmes et que les services gouvernementaux ont recours, dans le cadre de leurs activités courantes, à l'expertise générée par le programme du CRSNG. Ces résultats ne concordent qu'en partie avec ceux de Chapman et Farina (1983), qui indiquaient que les ministères ont des clientèles relativement différentes et qu'ils n'exploitent pas l'expertise développée dans le cadre du programme thématique du CRSNG. Cette différence s'explique probablement par le fait que notre étude porte sur dix années alors que la leur ne pouvait se fonder que sur trois années d'existence du programme thématique.

\section{LA PRODUCTION SCIENTIFIQUE EN ÉNERGIE SOLAIRE}

De 1974 à 1987, 154 articles portant sur l'énergie solaire ont été publiés par des chercheurs canadiens, dans les universités, les laboratoires publics et l'industrie, 
Tableau 6

Relations entre les deux programmes et les publications

17 chercheurs d'EMR:

aussi CRSNG

aussi publications
Obtenant plus de $200000 \$$ d'EMR

$2 / 6$

$3 / 6$
Tous les chercheurs

$5 / 17$

$6 / 17$

37 chercheurs principaux du CRSNG:

Obtenant plus de $200000 \$$ du CRSNG

$1 / 10$

$4 / 10$

Tous les chercheurs

$5 / 37$

$18 / 37$

aussi EMR
aussi publications

46 co-chercheurs du CRSNG:

aussi EMR

aussi publications

Tous les chercheurs

$2 / 46$

$9 / 46$

154 chercheurs ayant publié dans les 8 revues:

Ayant publić au moins 3 articles

$4 / 23$

aussi EMR

aussi CRSNG

$11 / 23$

Tous les chercheurs $6 / 154$

$18 / 154$

Sources: CRSNG: données compilées. à partir des rapponts annuels; EMR: données compilées à partir du Bulletin Recherche el développement: publications: à partir des huit revues en solaire.

dans les huit revues retenues, soit une moyenne de 11 articles par année. On les retrouve surtout dans Solar Energy (94), Solar Energy Materials (21), Journal of Applied Physics (17) et Journal of the Electrochemical Society (11). Comme l'indique le tableau 7, la production canadienne en énergie solaire s'est accrue en trois phases. La première se termine en 1979 et est caractérisée par un accroissement important du nombre d'articles publiés par les canadiens. La deuxième période, de 1979 à 1982, est marquée par une relative stabilité, alors que la troisième, de 1983 à 1987, est aussi stable, mais à un niveau plus élevé. Cette tendance suit la croissance de la communauté scientifique internationale en énergie solaire, qui amène la création de nouvelles revues, comme Solar Cells et Solar Energy Materials en 1979 (Leydesdorff et van der Schaar, 1987). En somme, cette période voit la consolidation d'une nouvelle spécialité. Ainsi, la Solar Energy Society of Canada Inc. tient un congrès annuel depuis 1975 et en publie les actes depuis 1977. À la même époque, d'autres lieux de rencontre apparaissent aux États-Unis, comme la Division d'énergie solaire de l'American Society of Mechanical Engineers (1978) et le Symposium annuel sur les cellules photovoltaiques de l'Electrochemical Society inauguré en 1979.

La communauté canadienne des chercheurs en énergie solaire, telle qu'elle ressort de ces 154 articles, est composée de 192 chercheurs. La majeure partie provient des universités (154), alors que les autres sont associés à l'industrie (20) et à des centres de recherche gouvernementaux (18). Trente chercheurs ont publié au moins trois articles parmi lesquels 23 sont à l'université, 3 dans l'industrie et 4 dans des laboratoires gouvernementaux. Ainsi, quelques institutions hors du 
Tableau 7

Nombres d'articles publiés par des Canadiens par année en solaire

a nnée

nombre

moyenne-mobile ( 3 ans)

1974

1975

1976

1977

1978

1979

1980

1981

1982

1983

1984

1985

1986

1987

(1)

1

4

3

5

11

17

9

10

16

10

20

10

18

17

-
3
4
8
11
12
13
12
12
15
13
16
15
-

Source: à partir des huit revues en solaire.

réseau universitaire apparaissent des producteurs importants de publications dans le domaine. Les chercheurs universitaires qui publient sur l'énergie solaire proviennent surtout de quatre disciplines : chimie, physique, génie électrique et génie mécanique (tableau 3 ).

Dans 63\% des cas, les auteurs indiquent la provenance de leurs subventions, ce qui nous a permis d'identifier plusieurs organismes (tableau 8). Les plus importants sont le CRSNG (pour ses programmes généraux et thématiques), le CNR (pour ses différents programmes de recherche) et le ministère fédéral de l'Environnement. Les remerciements ne sont cependant pas suffisamment précis pour qu'il soit possible de distinguer les divers programmes du CRSNG. En ce qui concerne le CNR, les Directions ne sont pas non plus toujours indiquées. Les Directions mentionnées sont l'énergie, la chimie et la construction.

Les deux programmes retenus figurent effectivement parmi les plus importants : le CRSNG est le premier, suivi des programmes en énergie du CRN et d'EMR. Ces deux sources ne couvrent toutefois que les deux tiers des remerciements. Des fonds sont disponibles de plusieurs autres organismes et, dans $13 \%$ des cas, les organismes impliqués ne sont pas canadiens : il s'agit d'institutions internationales ou américaines qui ont fortement soutenu la recherche en énergie solaire.

Le financement provient donc presque exclusivement des gouvernements, mais de sources très variées, preuve que plusieurs organismes sont impliqués dans le soutien à la recherche en énergie solaire. L'industrie semble très peu impliquée dans le financement de la recherche, quoiqu'elle réalise elle-même une partie de la recherche à partir de ses propres fonds. 
Tableau 8

Organismes subventionnaires mentionnés dans les articles

\begin{tabular}{lcc}
\hline Organisme subventionnaire & nombre & $\%$ \\
\hline CRSNG & 61 & 51 \\
CNR & 16 & 13 \\
EMR & 2 & 2 \\
Environnement & 7 & 6 \\
CNR (programme PARI) & 3 & 3 \\
Autres fédéraux & 1 & 1 \\
Organismes américains & 8 & 7 \\
Autres pays & 4 & 3 \\
OrAN & 4 & 3 \\
& & 2 \\
Industrie & 2 & 2 \\
Universités & 2 & 2 \\
FCAR & 2 & 4 \\
Colombie-Brit. & 5 & 3 \\
autres provinces & 3 & 100 \\
Total: & 120 &
\end{tabular}

Source: à partir des huit reyues en solaire.

\section{LES RELATIONS ENTRE LES SUBVENTIONS ET LES PUBLICATIONS}

La figure 1 présente les liens entre les deux programmes de subvention et les publications. La relation la plus forte unit les chercheurs ayant publié et ceux ayant obtenu des subventions du CRSNG - action thématique en énergie. En effet, la moitié des chercheurs principaux ont aussi publié dans les principales revues consacrées à ce domaine. Ces résultats montrent que le programme tend à financer les chercheurs les plus actifs dans le domaine.

Les co-chercheurs montrent un profil assez différent. Moins de $20 \%$ des co-chercheurs ont publié dans les huit revues, comparativement à $50 \%$ pour les chercheurs principaux. Ces résultats indiquent que les co-chercheurs sont beaucoup moins impliqués dans les projets de recherche. Dans les trois plus grandes équipes, deux des trois chercheurs principaux, mais aucun des 21 co-chercheurs, ont publié dans l'une ou l'autre des huit revues retenues ici, ce qui est le cas du tiers des chercheurs subventionnés par EMR.

Seulement trois chercheurs figurent sur les trois listes. Ils sont très actifs dans le domaine : un a publié au moins 3 articles et obtenu plus de 200000 d'EMR : un autre a obtenu plus de $200000 \$$ d'EMR et le troisième a publié au moins trois articles. 


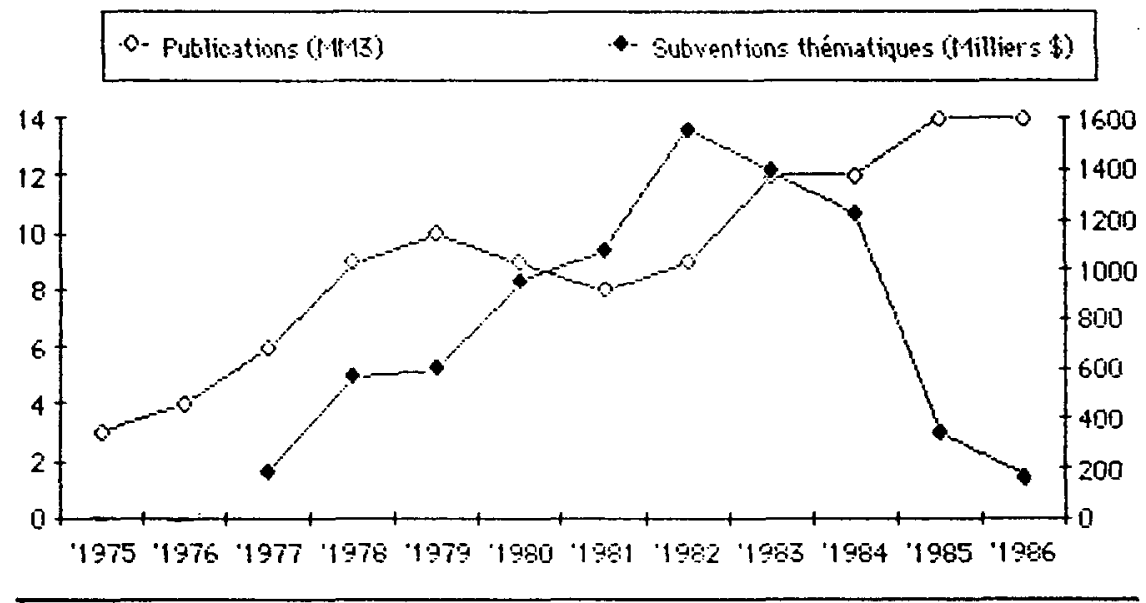

Sur les 192 chercheurs, 154 sont des universitaires. Un bon nombre sont probablement des étudiants. Vingt-trois chercheurs universitaires, ayant publié au moins 3 articles, au cours de la période étudiée, forment le centre de la communauté des chercheurs actifs en énergie solaire. Dix d'entre eux n'ont pas obtenu de contrats d'EMR ou de subventions thématiques du CRSNG, mais huit bénéficiaient de subventions ordinaires du CRSNG ; deux ont obtenu du financement des deux programmes, deux d'EMR seulement et neuf du CRSNG seulement. Parmi les dix chercheurs admissibles au programme des subventions thématiques du CRSNG, seulement la moitié en ont bénéficié. Le CRSNG ne donnant pas accès aux dossiers, il n'est malheureusement pas possible de connaître la proportion de ceux qui ont effectivement soumis une demande. Bien que seulement deux chercheurs aient obtenu des fonds d'EMR, il est possible que d'autres chercheurs aient obtenu des contrats d'autres ministères fédéraux, comme l'indiquent les données sur les remerciements.

Afin de savoir si les chercheurs étaient actifs dans la recherche en énergie solaire avant d'obtenir des fonds du programme thématique du CRSNG, on a regardé s'ils ont publié ou non dans le domaine avant d'obtenir des subventions de cet organisme. Dans un premier temps on présente les tendances globales, pour ensuite regrouper les chercheurs en fonction des publications produites et des subventions reçues. Les données des programmes d'EMR ne couvrant que la fin de la période étudiée, il n'est pas possible de les analyser selon ces critères.

Durant les premières années d'existence du programme thématique en énergie du CRSNG, on remarque un accroissement simultané du nombre de publications dans le champ de l'énergie solaire et du financement obtenu (figure 2). Les données indiquent cependant que l'augmentation des publications est antérieure à l'octroi des subventions. Ce fait indique qu'un groupe de chercheurs était déjà actif 
dans la recherche en énergie solaire avant la mise en place du programme de subvention. Au cours des dernières années, le financement décroît considérablement, alors que le volume des publications demeure relativement élevé. Il est trop tôt cependant pour évaluer l'effet à plus long terme de cette réduction des budgets alloués à la recherche en énergie solaire. Les scientifiques vont-ils changer de domaine, revenir à leurs activités antérieures ou continuer leurs recherches à l'aide des programmes de subvention ordinaire du CRSNG? Bien que les subventions thématiques du CRSNG ne représentent pas la totalité des subventions accordées au secteur de l'énergie solaire, la courbe des subventions thématiques en énergie solaire reflète la tendance globale à la diminution au cours des cinq dernières années.

Seulement six des dix-huit chercheurs ayant publié dans les revues retenues et qui ont obtenu du financement thématique du CRSNG ont publié avant ou la même année que l'attribution de leur première subvention. Quatre d'entre eux bénéficiaient alors de subventions ordinaires pour des projets reliés de près à l'énergie solaire. Pour les douze autres chercheurs, la comparaison des thèmes de leurs subventions ordinaires et stratégiques permet de conclure qu'il s'agit plutôt d'une application particulière d'un programme de recherche plus général. Par exemple, un chercheur intitule sa subvention ordinaire «Physical chemistry of surfaces" et sa subvention thématique «Direct conversion of solar energy ». Un autre passe de «solid state electronics» à «Solar energy» etc. Enfin, il est intéressant de constater que parmi les dix-neuf chercheurs qui n'ont pas publié dans les revues spécialisées en énergie solaire, aucun n'avait une activité de recherche reliée de près à ce secteur. D'autre part, parmi les douze chercheurs qui ont publié au moins trois articles dans les revues d'énergie solaire et qui n'ont toutefois pas obtenu de subventions thématiques, dix ont bénéficié de subventions ordinaires dont cinq pour des projets consacrés à l'énergie solaire et deux à la météorologie.

\section{CONCLUSION}

Les différents programmes de subvention et de contrats à la recherche en énergie visaient à accroître le niveau de la recherche orientée dans des domaines jugés d'intérêt national. L'étude du nombre de publications canadiennes dans le champ de l'énergie solaire a montré un accroissement de l'activité de recherche dans ce secteur. En effet, alors que le niveau des publications était très faible en 1974, il augmente rapidement pendant quelques années pour ensuite se maintenir à un niveau relativement élevé jusqu'à la fin de la période étudiée. Près de 200 chercheurs appartenant aux secteurs industriel, gouvernemental et universitaire ont contribué à ces publications.

Plusieurs facteurs peuvent expliquer cette croissance des activités de recherche. Le premier est la création d'une communauté de chercheurs dans le domaine, marquée par le développement d'institutions (comme des associations et des congrès) et par la création de revues spécialisées qui sont devenues les lieux de publication privilégiés par les chercheurs. La communauté est formée surtout de 
chercheurs universitaires, mais comprend aussi des chercheurs des laboratoires publics et des entreprises. Bien que ces derniers aient publié plusieurs articles, l'industrie contribue très peu au financement de la recherche universitaire.

Un autre facteur important est évidemment l'apport des organismes subventionnaires. Cette nouvelle communauté a réussi à obtenir beaucoup de financement pour réaliser ses projets de recherche. En ce qui concerne le programme thématique en énergie du CRSNG, les chercheurs actifs en solaire se retrouvaient en concurrence avec les autres domaines et ils ont obtenu une fraction significative du financement, se classant au premier rang avec $16,3 \%$ du financement total, suivi de la fusion $(15,3 \%)$ et de la conservation d'énergie $(12,6 \%)$. À EMR, les chercheurs étaient en concurrence avec les entreprises et les laboratoires publics et c'est dans le domaine de l'énergie solaire que les chercheurs universitaires ont obtenu la plus large part du financement, soit $31,5 \%$. On a aussi signalé un grand nombre d'organismes publics qui ont donné des fonds aux chercheurs canadiens.

On peut supposer que l'attribution des fonds se fait davantage selon la force des communautés scientifiques que selon la pertinence socio-économique des projets; les communautés déjà formées ayant la capacité de canaliser des ressources importantes et ce selon leurs propres intérêts scientifiques et institutionnels. Dans le programme stratégique du CRSNG, par exemple, il est curieux que les chercheurs en solaire aient obtenu deux fois plus de fonds que les chercheurs en biomasse et trois fois plus que ceux travaillant sur les sables pétrolifères, domaines qui correspondent pourtant davantage aux caractéristiques de l'économie canadienne.

Le rôle des organismes subventionnaires dans la croissance du secteur de l'énergie solaire demeure difficile à estimer avec précision. Nos résultats ont indiqué, premièrement, que les deux sources étudiées étaient importantes, mais qu'il y avait aussi plusieurs autres sources de fonds, dont les subventions ordinaires du CRSNG, qui demeurent la première source pour les chercheurs universitaires, et les contrats de recherche offerts par un grand nombre d'organismes publics. Deuxièmement, la communauté était déjà en émergence au moment de la création des programmes de recherche orientée et plusieurs chercheurs publiaient dans le domaine avant d'obtenir des fonds du programme thématique. Cela suggère que ces programmes, en canalisant vers les chercheurs en solaire des fonds importants pendant une courte période, ont pu intensifier les recherches dans le domaine.

Les résultats de notre recherche nous permettent de classer les chercheurs qui font partie de notre population en cinq groupes distincts. Le premier regroupe six chercheurs qui étaient actifs dans le domaine de l'énergie solaire avant la création des programmes thématiques et d'EMR, qui ont publié et qui ont obtenu une ou des subventions du programme thématique du CRSNG. Presque tous ont aussi bénéficié de contrats d'EMR. On peut dire que, pour ce groupe, les programmes ont contribué à intensifier leur activité de recherche. Le second, de même taille que le premier, est composé de chercheurs qui étaient actifs dans le domaine mais qui n'ont pas participé au programme thématique du CRSNG. Le troisième groupe 
réunit douze chercheurs qui ont publié après avoir reçu des subventions thématiques mais qui travaillaient jusque là dans des secteurs connexes à l'énergie solaire et qui ont adapté leur programme de recherche pour faire place à ce thème et profiter ainsi des nouveaux programmes. C'est ce groupe qui répond le mieux aux objectifs du programme du CRSNG qui visait à encourager les chercheurs à entrer dans le domaine de l'énergie solaire. Enfin, le quatrième groupe comprend dix-neuf chercheurs qui n'ont pas publié et dont les sujets de recherche n'étaient pas vraiment reliés au domaine de l'énergie solaire mais qui ont toutefois bénéficié de subventions thématiques. Il est possible, cependant, que ces chercheurs aient publié dans un domaine connexe à l'énergie solaire non couvert par les principales revues retenues dans notre étude. Un dernier groupe comprend douze chercheurs qui obtiennent des contrats d'EMR mais ne bénéficient pas de subventions thématiques. Il s'agit de chercheurs qui publient peu. Comme on l'a vu plus haut il s'agit surtout de chercheurs en génie dont plusieurs sont affiliés aux centres d'expertise en énergie solaire. Les universités étant plus actives dans leur recherche de fonds de nature contractuelle, ce groupe, davantage tourné vers les applications pratiques, est celui dont les caractéristiques correspondent le mieux au type de comportement que requiert ce genre de recherche contractuelle. À une époque où les relations université-industrie prennent une importance croissante, il serait intéressant d'analyser les modes d'insertion de ces chercheurs au sein de l'université (tant en termes de publications que d'enseignement aux cycles supérieurs) et de leurs communautés scientifiques respectives et de les comparer à ceux des chercheurs qui vivent surtout de subventions traditionnelles.

L'existence des deuxième et quatrième groupes de chercheurs porte à croire que les critères et mécanismes d'attribution des subventions n'arrivaient pas vraiment à distinguer les chercheurs vraiment actifs dans le domaine de ceux qui adaptaient de façon ad hoc leurs projets de recherche pour bénéficier temporairement de nouvelles sources de financement : en effet, dans le premier cas, on a affaire à des chercheurs actifs en solaire qui n'ont pas participé aux actions thématiques, alors que dans le second on retrouve des chercheurs non actifs en solaire qui ont bénéficié du programme. La diminution des ressources consacrées à l'énergie solaire par le gouvernement fédéral, risque d'affecter davantage les membres du troisième groupe qui choisiront probablement de retourner à leur programme initial de recherche dont la nature assez générale permet une adaptation en fonction de différentes applications. Ils se tourneront alors vers d'autres sujets devenus «prioritaires » pour profiter des subventions offertes.

Nos données suggèrent qu'il n'est pas facile de réorienter les activités des chercheurs même lorsque ceux-ci mordent à l'hameçon des nouveaux programmes. Un exemple extrême est peut-être ce biologiste qui après avoir fait «travailler» ses bactéries pour produire de l'énergie solaire, s'est tourné vers le programme de biotechnologie pour leur faire produire de l'ammoniac ... Pour parvenir à réorienter l'activité des chercheurs en fonction de besoins «nationaux » ou d'objectifs jugés prioritaires, les gouvernements auraient tout intérêt à tenir compte de tous les facteurs affectant le choix des projets par les chercheurs. 
L'autonomie relative des communautés scientifiques étant difficilement contournable, les gouvernements devront y prêter une plus grande attention s'ils veulent véritablement avoir prise sur la dynamique de la recherche.

\section{BIBLIOGRAPHIE}

Berkowitz, M.K. (1978), "A Review of Canadian and US Solar Energy Policies ", Canadian Public Policy, vol. 2, no. 2, p. 257-262.

Chapman, I.D. et C. Farina (1981), «An Analysis of Some Problems in Re-orientating University Research ", Canadian Public Policy, vol. 7, no. 2, pp. 297-305.

Chapman, I.D. et C. Farina (1983), «Peer Review and National Need», Research Policy, vol. 12, pp. 317-327.

CNR (1986), Technologie des énergies de remplacement au Canada - Programme de $R$ et $D$ énergétiques du CNRC, 1975-1985.

CRSNG (1985), Un deuxième plan quinquennal pour les programmes du Conseil de recherche en sciences naturelles et en génie. Préparer la voie vers les années 1990, Ottawa, juin.

Enermodal Engineering (1979), Solar Thermal Technology Report, Toronto, Ontario Ministry of Energy.

Ford, Glyn, Ken Green, Fred Steward et Salma Ahmad (1986), «Co-ordinating Strategic Research in Britain », Science and Public Policy, vol. 13, no. 5, pp. 269-274.

Gingras, Yves et Jacques Rivard (1988), «R\&D Energy Policy in Canada», Science and Public Policy, vol. 15, no. 1, pp. 35-42.

Irvine, John, Ben R. Martin et Geoffrey Oldham (1983), Research Evaluation in British Science : A SPRU Review, Science Policy Research Unit, University of Sussex, Commissioned by the Centre de prospective et d'évaluation, Ministère de la recherche et de l'industrie (France).

Leydesdorff, Loe et Peter van der Schaar (1987), «The Use of Scientometric Methods for Evaluating National Research Programs ", Science \& Technology Studies, vol. 5, no. 1, pp. 22-31.

Rip, Arie and Madeli Hennekam (1985), Acknowledging Funding in Biotechnology and Bioengineering, National Science Foundation.

Roy, R. (1984), «Alternatives to Review by Peers: a Contribution to the Theory of Scientific Choice», Minerva, vol. 22, pp. 316-328. 